\title{
Effect of EDTA treatment on the hybrid layer durability in total-etch dentin adhesives
}

\author{
Duck-Su KIM${ }^{1}$, Sang Hyuk PARK ${ }^{1,2}$, Gi-Woon $\mathrm{CHOl}^{1}$, Kyoung-Kyu CHOI${ }^{1}$ and Sun-Young $\mathrm{KIM}^{1}$ \\ ${ }^{1}$ Department of Conservative Dentistry, School of Dentistry, Kyung Hee University, Hoegi-dong, Dongdaemun-gu, Seoul, Korea, 130-702 \\ ${ }^{2}$ Department of Conservative Dentistry, Dental Hospital, Kyung Hee University Hospital at Gangdong, Seoul, Korea,134-727 \\ Corresponding author, Sun-Young KIM; E-mail: kimsunyoung@khu.ac.kr
}

\begin{abstract}
The effect of the ethylenediaminetetraacetic acid (EDTA) treatment on the hybrid layer durability of total-etch dentin adhesives was investigated. A flat dentin surface was exposed and treated with $37 \%$ phosphoric acid or 0.1M EDTA. Dentin adhesive was applied and a $4 \mathrm{~mm}$ thick composite resin was built-up. Twenty $0.9 \times 0.9 \mathrm{~mm}^{2}$ resin-dentin beams were fabricated in each experimental group. Artificial aging with $10 \% \mathrm{NaClO}$ was performed in half of the experimental groups for 1 hour. A micro-tensile bond strength ( $\mu$ TBS) test was performed and the bonded interface was analyzed with transmission electron microscopy (TEM). The $37 \%$ phosphoric acid-treated group showed a lower $\mu$ TBS after artificial aging, whereas the 0.1M EDTA-treated group did not. TEM analysis revealed collagen fibrils dissolved in the $37 \%$ phosphoric acid-treated group but not in the $0.1 \mathrm{M}$ EDTA-treated group. Undissolved minerals were observed in the 0.1M EDTA-treated group. In conclusion, a dentin treatment with 0.1M EDTA is effective in improving the hybrid layer durability.
\end{abstract}

Keywords: EDTA, Hybrid layer,Total-etch dentin adhesive, $\mu$ TBS, TEM, Accelerated aging

\section{INTRODUCTION}

Although the immediate resin-dentin bond strength has been increased by the development of a total-etch adhesive system, the long-term durability of a resin-dentin bond is still a major challenge in adhesive dentistry ${ }^{1}$. Ideally, the exposed collagen fibrils after acid-etching should be embedded completely by the adhesive resin monomers to form an optimally sealed hybrid layer. However, the homogeneous infiltration of adhesive resin monomers through the exposed interfibrillar space barely occurs due to several factors, such as histological complexity of the dentin matrix and collapse of the exposed collagen fibrils after air-drying ${ }^{2,3)}$. The resulting unprotected collagen fibrils are prone to degrade rapidly within the hybrid layer over time, which acts as a weak point of the resin-dentin bond. Several in vivo and in vitro studies examining the durability of the adhesive interface have indicated that the hybrid layer degrades rapidly when the adhesive interface is exposed to oral fluids or artificial saliva after only a few months of clinical application or storage ${ }^{4,5}$.

Ethylenediaminetetraacetic acid (EDTA) has the ability to remove the smear layer formed on the dentin surface after tooth preparation as well as to demineralize dentin by chelating calcium ions. EDTA has been used widely to dissolve the mineral phase of dentin while avoiding major alterations of the native fibrillar structure of the collagen in dentin ${ }^{6,7)}$. Cederlund et al. reported that an EDTA treatment increased immediate shear bond strength ${ }^{8)}$. Sauro et al. also reported that conditioning of the smear layers with EDTA produced a less porous resin-dentin interface ${ }^{9}$. These studies reported that an EDTA treatment of the dentin surface has a favorable effect on the resin-dentin bond.
Nevertheless, there are few reports on the effect of EDTA on the bonding durability.

Among the artificial aging methods used to test the durability of the resin-dentin bond in vitro, the use of an aqueous solution of sodium hypochlorite $(\mathrm{NaClO})$ as a storage medium has been suggested to be suitable for assessing the durability of the hybrid layer ${ }^{10)} \cdot \mathrm{NaClO}$ induces the degradation of suboptimally protected collagen fibrils caused by incomplete resin infiltration into the demineralized dentin layer. A previous study reported that when a resin-dentin bond was stored in $10 \% \mathrm{NaClO}$ aqueous solution, degradation of the collagen fibrils within the demineralized dentin or hybrid layer occurred according to scanning electron microscopy (SEM) and transmission electron microscopy (TEM), which caused a decrease in resin-dentin bond strength ${ }^{11}$.

This study examined the effect of an EDTA treatment on the hybrid layer after artificial aging with $10 \% \mathrm{NaClO}$ aqueous solution. The bonded interfaces were also examined by TEM. The null hypothesis of this study was that there is no difference in the microtensile bond strength of the resin-dentin bond between the $37 \%$ phosphoric acid treatment and 0.1M EDTA treatment irrespective of artificial aging with $10 \% \mathrm{NaClO}$ aqueous solution.

\section{MATERIALS AND METHODS}

\section{Specimen preparation}

Twelve caries-free third molars were used. The protocol of this study was approved by the Institutional Review Board (IRB) committee of Kyung Hee Medical Center (KHB IRB 003-2). The occlusal enamel was removed and the flat dentin surface was exposed. This surface was polished with 320-grit silicon carbide abrasive paper to 
produce a standard smear layer. The teeth were divided randomly into four experimental groups.

For the dentin treatment, half of the specimens were treated with $37 \%$ phosphoric acid aqueous solution (Scotchbond Etchant, 3M ESPE, St. Paul, USA) for 15 seconds before the adhesive procedure, and the other half of the specimens were treated with $0.1 \mathrm{M}$ EDTA aqueous solution (Sigma-Aldrich, St. Louis, USA) for 60 seconds. 37\% Phosphoric acid- or 0.1 M EDTA-treated dentin surfaces were rinsed with water for 10 seconds and excess water on the dentin surface was blot-dried. The total-etch dentin adhesive (Adper Single Bond 2, 3M ESPE) was applied to the dentin surface and light-cured for 20 seconds. A hybrid composite (Clearfil AP-X, Kuraray Medical, Sakazu Kurashiki, Okayama, Japan) was then placed incrementally with a $2 \mathrm{~mm}$ thickness to make a $4 \mathrm{~mm}$ height. Each composite layer was light-cured for 40 seconds. A QTH (Quartz Tungsten Halogen) light curing unit (VIP Junior, Bisco Inc., Schaumburg, USA) with an intensity of $600 \mathrm{~mW} / \mathrm{cm}^{2}$ was used. The specimens were then stored in distilled water at $37^{\circ} \mathrm{C}$ for 24 hours. The composition of Adper Single Bond 2 was listed in Table 1.

Artificial aging and micro-tensile bond strength test After 24 hours storage, each tooth-composite bonded specimen was sectioned occluso-gingivally into $0.9 \mathrm{~mm}$ thick serial slabs using a low-speed diamond saw (Isomet, Buehler Ltd., IL, USA). These slabs were sectioned further into $0.9 \times 0.9 \mathrm{~mm}^{2}$ composite-dentin beams according to the technique for the non-trimming version of the micro-tensile bond strength test ${ }^{12)}$. Compositedentin beams of either $37 \%$ phosphoric acid- or $0.1 \mathrm{M}$ EDTA-treated specimens were divided into two sub-groups: no aging group and artificial aging group (Table 2). Twenty composite-dentin beams were assigned to each experimental group. The composite-dentin beams of the artificial aging group were immersed in a $10 \%$ $\mathrm{NaClO}$ aqueous solution $(\mathrm{pH}$ 12.80; Sigma-Aldrich, St. Louis, USA) for 1 hour $^{13)}$. Each composite-dentin beam was mounted on a $\mu$ TBS testing jig with cyanoacrylate adhesive (Zapit, Dental Ventures of America, USA) and a $\mu$ TBS test was performed using a universal testing machine (EZ-Test, Shimadzu, Japan) at a crosshead speed of $1.0 \mathrm{~mm} /$ minute.

\section{Transmission electron microscopy analysis}

The specimens for TEM analysis were prepared according to the protocol reported by Tay et al. ${ }^{14}$. Two teeth from each group were selected. Each dentin treatment and composite build-up were performed the same way as that used for the preparation for the $\mu$ TBS test. A $0.9 \mathrm{~mm}$ thick slab was sectioned with a low-speed diamond saw. Two central slabs were selected for each tooth so that four slabs were used for each group. Two coats of nail varnish were applied, leaving $1.0 \mathrm{~mm}$ from the resin-dentin interface. Each slab was immersed in a 50 wt\% ammoniacal silver nitrate solution for 24 hours in a dark room and washed with distilled water. The samples were then stored in a developing solution for 8 hours under fluorescent light and washed with distilled water. Each slab was trimmed to a $6 \mathrm{~mm}$ width and fixed in Karnovsky's solution [2.5\% glutaraldehyde and 2\% paraformaldehyde in $0.1 \mathrm{M}$ sodium phosphate buffer $(\mathrm{pH}$ 7.4)] for 8 hours, and then washed 3 times for 10 minutes with $0.1 \mathrm{M}$ sodium phosphate buffer solution ( $\mathrm{pH} 7.4$ ). Post-fixation was performed with a $1 \%$ osmium tetroxide solution for 2 hours. Each slab was washed 3 times for 10 minutes each. The post-fixed specimen was dehydrated in a graded series of ethanol $(50,70,80,90,95$, and $100 \%$ ) for 10 minutes and embedded in epoxy resin. After screening a $200 \mathrm{~nm}$ semi-thin section of each specimen using an ultra-microtome equipped with a diamond knife, an approximately $70 \mathrm{~nm}$ ultra-thin section was cut and collected on 75-mesh formvar-coated copper grid. After drying, the samples were examined by TEM (JEM-1010, JEOL Ltd., Tokyo, Japan) operating at 75 $\mathrm{kV}$.

\section{Statistical analysis}

The data was analyzed by two-way ANOVA to find significant differences between the dentin surface

Table 1 The composition of Adper Single Bond 2 used in this study

\begin{tabular}{lll}
\hline Adhesive System & \multicolumn{1}{c}{ Composition } & Manufacturer \\
\hline & $\begin{array}{l}\text { Water, ethanol, HEMA, Bis-GMA, dimethacrylates, initiators, } \\
\text { methacrylate functional copolymer of polyacrylic and polyitaconic acids, }\end{array}$ & $\begin{array}{l}\text { 3M ESPE, } \\
\text { Adper Single Bond } 2\end{array}$ \\
& $\begin{array}{l}\text { and silica nanofillers } \\
\text { Etching: 37\% phosphoric acid }\end{array}$ & USA \\
\hline
\end{tabular}

Table 2 Experimental groups

\begin{tabular}{llr}
\hline Surface treatment & Aging treatment & Group code \\
\hline $\begin{array}{l}37 \% \text { phosphoric acid } \\
\text { for } 15 \text { seconds }\end{array}$ & No aging & PA0 \\
\hline $0.1 \mathrm{M}$ EDTA for 60 seconds & Artificial aging & PA1 \\
\hline
\end{tabular}


treatment and aging treatment. Duncan's multiple comparison tests were performed at the $95 \%$ significance level.

\section{RESULTS}

Microtensile bond strength test

Figure 1 shows the $\mu$ TBS of the four experimental groups. A significant difference was observed based on the aging treatment (no aging vs. artificial aging, $p<0.05$ ) but not on the dentin surface treatment (37\% phosphoric acid vs. $0.1 \mathrm{M}$ EDTA, $p>0.05$ ). The interaction between these two factors was also significant $(p<0.05)$. In the $37 \%$ phosphoric acid-treated group, the artificial aging sub-group showed a lower mean $\mu$ TBS than the sub-group without aging $(p<0.05)$. In the $0.1 \mathrm{M}$ EDTA-treated group, there was no significant difference in the mean $\mu$ TBS between the sub-groups with and without the aging treatment $(p>0.05)$.

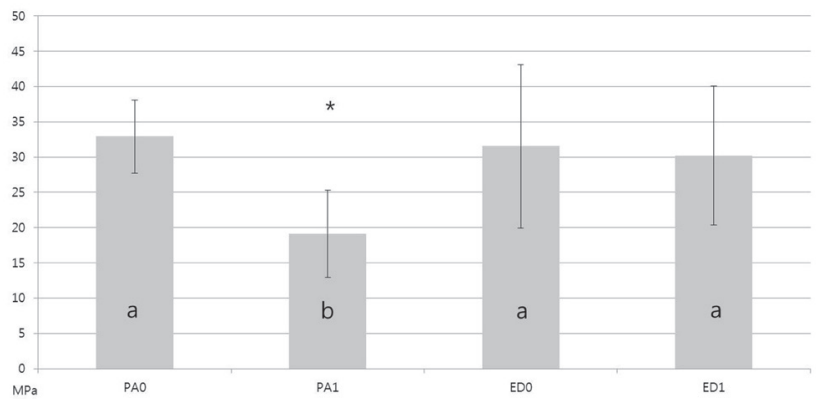

Fig. 1 Micro-tensile bond strength of four experimental groups. Groups with the same letters in the bar indicate no significant difference $(p<0.05)$.

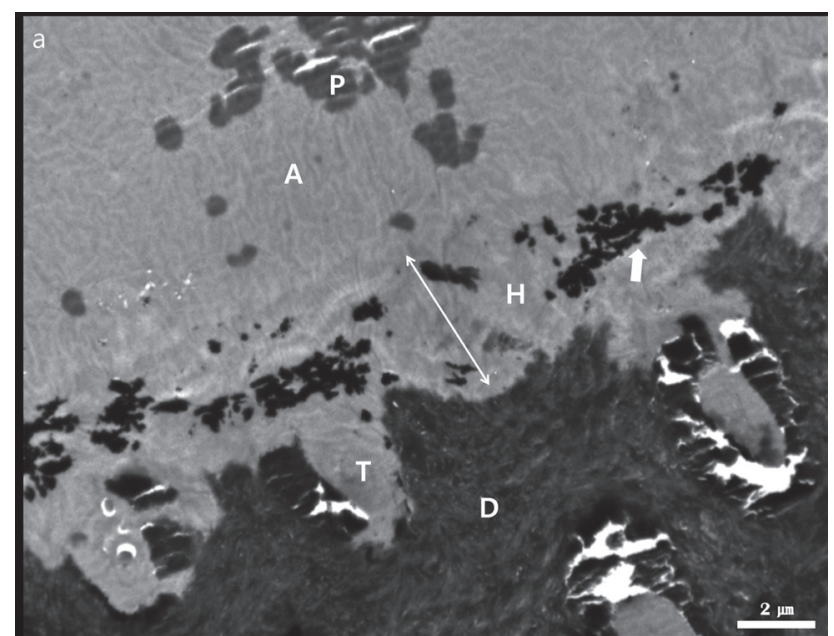

Transmission electron microscope analysis

Figures 2-5 show TEM images of all experimental groups. The $37 \%$ phosphoric acid-treated groups showed relatively thick hybrid layer formation of $4-5 \mu \mathrm{m}$ and apparent resin tags formation into the dentinal tubule. However, deposition of many silver particles was observed in the hybrid layer, which suggested that collagen fibrils were not completely encapsulated or

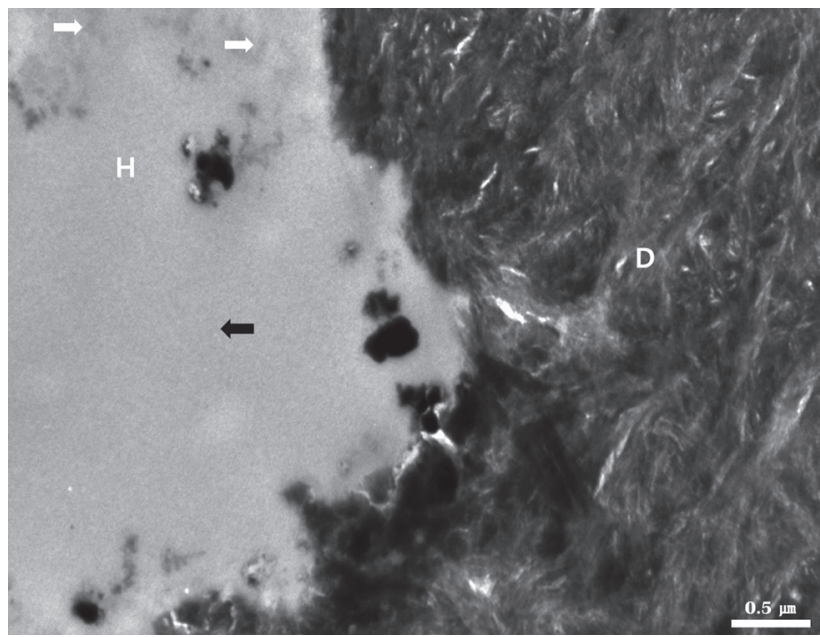

Fig. 3 TEM image of PA1 (original magnification: $\times 20,000$ ). The collagen fibrils were partially dissolved in the hybrid layer and seen as an electro-lucent area (black arrow). White arrowindicated area suggests that collagen fibrils were protected by adhesive resin infiltration from $\mathrm{NaClO}$ aging, which was seen as relatively electro-dense area in the hybrid layer. H: hybrid layer, D: dentin.

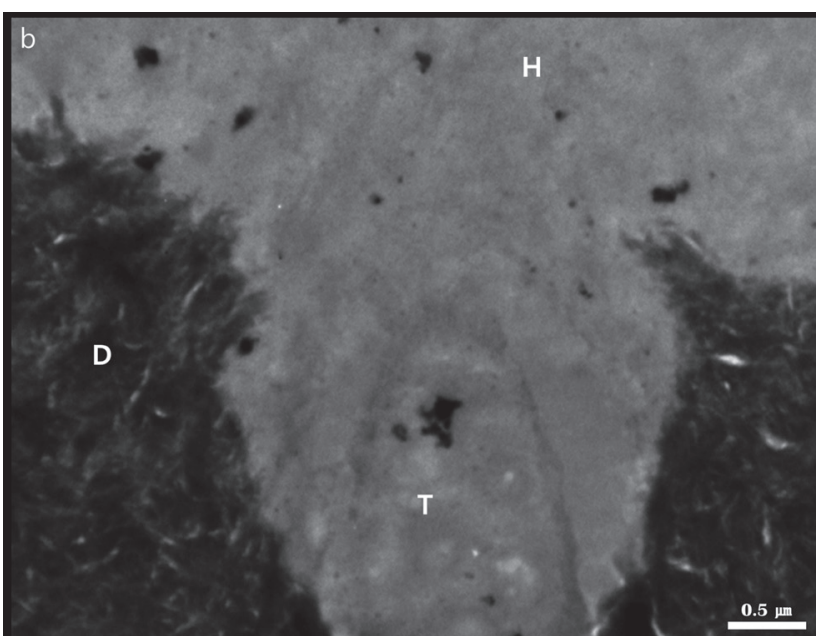

Fig. 2 (a) TEM image of PA0 (original magnification: $\times 5,000$ ). Apparent resin tags formation through the dentinal tubule were observed. Deposition of many silver particles appeared in the hybrid layer (white arrow). The thickness of hybrid layer was approximately 4-5 $\mu \mathrm{m}$ (white double arrow). P: polyalkenoic copolymer, A: adhesive resin, H: hybrid layer, T: resin tag, D: dentin. (b) TEM image of PA0 (original magnification: $\times 20,000$ ). The distinction between the dentin substrate and hybrid layer was obvious. H: hybrid layer, T: resin tag, D: dentin. 

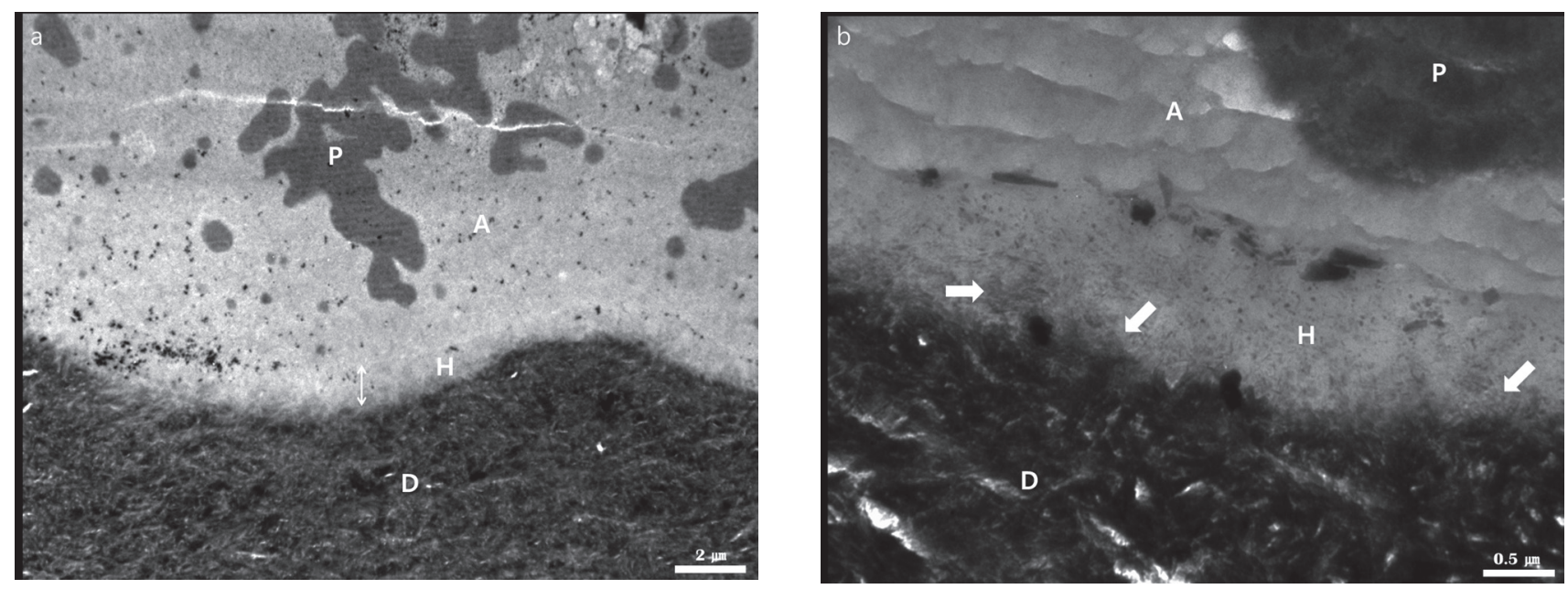

Fig. 4 (a) TEM image of ED0 (original magnification: ×5,000). Resin tags were rarely observed in the hybrid layer. The thickness of the hybrid layer was approximately 1-2 $\mu \mathrm{m}$ (white double arrow). P: polyalkenoic copolymer, A: adhesive layer, H: hybrid layer, D: dentin. (b) TEM image of ED0 (original magnification: $\times 20,000$ ). The bottom of the hybrid layer was relatively flat and undissolved minerals were observed at the top of the dentin surface (white arrows). P: polyalkenoic copolymer, A: adhesive layer, H: hybrid layer, D: dentin.

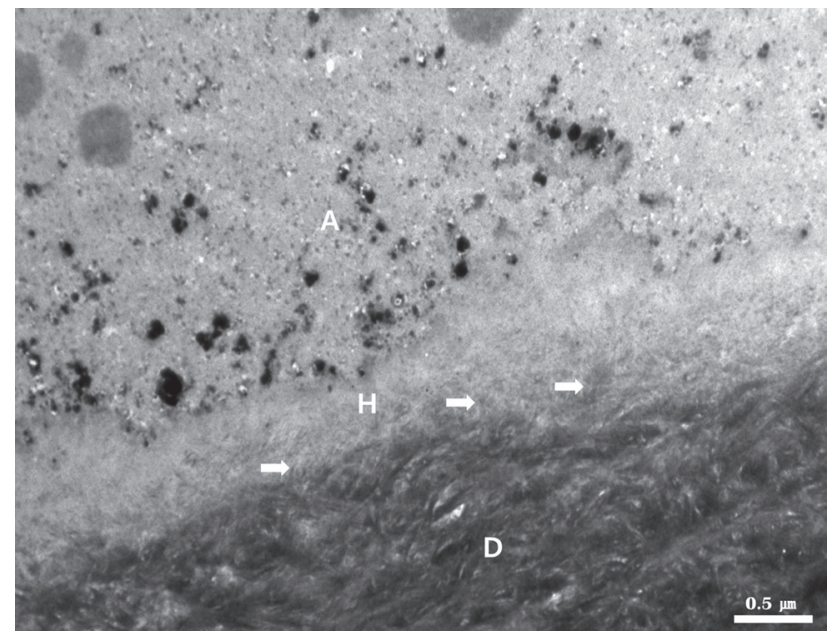

Fig. 5 TEM image of ED1 (original magnification: $\times 20,000)$. Dissolution of the collagen fibrils was not observed in the hybrid layer. Undissolved minerals were also observed at the top of the dentin surface (white arrows). A: adhesive layer, H: hybrid layer, D: dentin.

infiltrated by the adhesive resin and the hybrid layer was porous (Fig. 2a). The distinction between the dentin substrate and hybrid layer was obvious (Fig. 2b). In the aged specimens, the partial dissolution of collagen fibrils in the hybrid layer was reflected as relatively electrolucent area (Fig. 3).

As for the 0.1 M EDTA-treated groups, TEM images of ED0 and ED1 showed a different appearance compared to PA0 and PA1, respectively. Relatively thin hybrid layer of approximately 1-2 $\mu \mathrm{m}$ was formed and resin tags were rarely observed in the hybrid layer of EDTA-treated specimen compared to the $37 \%$ phosphoric acid-treated group (Fig. 4a). The bottom of the hybrid layer was relatively flat and undissolved minerals were observed at the top of the EDTA-treated dentin surface (Fig. 4b). The dissolution of the collagen fibrils was not observed even after artificial aging (Fig. 5).

\section{DISCUSSION}

In this study, a dentin treatment using 0.1 M EDTA showed a higher resin-dentin bond strength after artificial aging than that using 37\% phosphoric acid, which meaned an EDTA-treated resin-dentin interface had aging-resistant bond durability. Therefore, the null hypothesis of this study was rejected.

The total-etch dentin adhesive system has great advantages in terms of conditioning the enamel and dentin at the same time and giving high immediate dentin bond strength. However, issues of nanoleakage have been raised in unprotected collagen fibrils of the hybrid layer because the demineralization depth by the phosphoric acid is too deep for adhesive monomers to infiltrate the exposed collagen fibrils completely. The resin-dentin bond strength of the phosphoric acid-treated group decreased approximately $40 \%$ after artificial aging. This reduction is strongly believed to occur because $\mathrm{NaClO}$ deproteinizes the unprotected collagen fibrils in the bottom of the hybrid layer, which is infiltrated incompletely by the adhesive resin ${ }^{10,11)}$. TEM image also showed the dissolution of collagen fibrils in the bottom of the hybrid layer in this study (Fig. 3). The decrease in bond strength and the degradation aspect in TEM images after artificial aging in this study are 
similar to previous in vivo and in vitro studies, which reported the decreases in resin-dentin bond strength and collagen degradation in time or by other aging treatments including long-term water storage and thermocycling ${ }^{15-17)}$. It was recently reported that this nanoleakage due to a discrepancy between the depth of demineralization and the infiltration of adhesive resin might also occur not only for the total-etch adhesive but also for some self-etch adhesives ${ }^{18-20)}$.

The resin-dentin bonded interface treated with $0.1 \mathrm{M}$ EDTA showed strong resistance against artificial aging (Fig. 5). EDTA demineralizes dentin by chelation with calcium ions, so some minerals remain in the hybrid layer. The fact that the EDTA-treated resin-dentin bond strength did not decrease, even after artificial aging, suggests that there were few incompletely infiltrated collagen fibrils in the hybrid layer of the EDTA-treated resin-dentin interface. Moreover, the immediate bond strength of the EDTA-treated group did not show any differences from those of the phosphoric acid-treated group. It could be also speculated that EDTA treatment on the dentin causes strong hybrid layer despite its shallow demineralization depth. The strong bond strength of this EDTA-treated resin-dentin bond might be due to 3-phase hybridization between the collagen fibrils, adhesive monomer and partially demineralized hydroxyapatite bound to exposed collagen fibril, instead of the usual 2-phase hybridization of collagen fibrils and adhesive monomer ${ }^{6}$. Another possible explanation for the strong bond strength of the EDTA-treated resin-dentin bond in this study is that a Single Bond which contains polyalkenoic acid was used as the adhesive. The carboxylic group of polyalkenoic acid and the hydroxyapatite on the dentin surface or incompletely dissolved hydroxyapatite in the exposed collagen fibrils could form an ionic bond, which may have contributed to strong resin-dentin bond interface ${ }^{21)}$.

Considering that the demineralization effect of EDTA depends on the concentration and application time, the application of $0.1 \mathrm{M}$ EDTA for 60 seconds appears to be suitable for use clinically in terms of the immediate high bond strength and durable bonding. Erhardt et al. reported that the 0.1M EDTA-treated group showed a similar bond strength and higher dentin surface hardness when compared to the phosphoric acid-treated group ${ }^{22}$. Sauro also showed that the $0.1 \mathrm{M}$ EDTA-treated group showed no interfacial micropermeability and similar bond strength to the phosphoric acid-treated group ${ }^{9)}$. Osorio et al. reported that the 0.1M EDTA-treated group had a more durable resin-dentin bond after $\mathrm{NaClO}$ aging when compared to the total-etch dentin adhesive and self-etch dentin adhesive $^{23)}$. However, a separate enamel etching step and relatively long application time of 60 seconds could be a discouraging factor in clinics. Further studies will be needed on the concentration of EDTA capable of having the same effect with a shorter application time.

In this study, 1 hour immersion of the specimens in the $10 \% \mathrm{NaClO}$ aqueous solution, which is a recent in vitro aging treatment ${ }^{2)}$, was used to evaluate the resistance of the hybrid layer against degradation. $\mathrm{NaClO}$ is a non-specific deproteinizing agent that forms superoxide radicals and induces oxidation that fragments the long peptide chains in proteins. Moreover, it causes the chlorination of amino terminal groups and hypochlorous acid formation ${ }^{24)}$. Some of these amino-acid derived chloramines have also been shown to increase the proteolytic susceptibility of modified collagen fibrils $^{25)}$. Saboia et al. reported that the decrease in bond strength by artificial aging with $10 \% \mathrm{NaClO}$ for 1 hour was similar to that of 60,000 times thermocycling, which corresponds to approximately 6 years of aging ${ }^{13)}$. Considering this previous and the present studies showing an approximately $40 \%$ decrease in bond strength of the total-etch adhesive specimens after artificial aging, the immersion of the specimens in the $10 \% \mathrm{NaClO}$ solution for 1 hour would be suggested as a useful aging treatment simulating the in vivo aging.

This study showed that the use of $0.1 \mathrm{M}$ EDTA instead of $37 \%$ phosphoric acid minimized the incompletely resin-infiltrated collagen fibrils layer, giving the resin-dentin bond reliable durability. EDTA appears to be a good alternative dentin treatment prior to the application of an adhesive to make a durable resin-dentin bond in clinical situations because it could also inhibit the collagenolytic effect of matrix metalloproteinases by a chelation action ${ }^{26)}$. Additional studies, including a clinical study to confirm the possible use of EDTA in dental adhesion procedures, will be needed.

\section{ACKNOWLEDGMENTS}

This study was supported by Basic Science Research Program through the National Research Foundation of Korea (NRF) funded by the Ministry of Education, Science and Technology (NRF-20100023448).

\section{REFERENCES}

1) Breschi L, Mazzoni A, Ruggeri A, Cadenaro A, Di Lenarda R, Dorigo E. Dental adhesion review: aging and stability of the bonded interface. Dent Mater 2007; 24: 90-101.

2) Amaral FLB, Colucci V, Palma-Dibb RG, Corona Sa. Assessment of in vitro methods used to promote adhesive interface degradation : a critical review. J Esthet Restor Dent 2007; 19: 340-354.

3) De Munck J, Van Meerbeek B, Yoshida Y, Inoue S, Vargas M, Suzuki K, Lambrechts P, Vanherle G. Four-year water degradation of total-etch adhesives bonded to dentin. J Dent Res 2003; 82: 136-140.

4) Swift EJ Jr, Perdigao J, Heymann HO, Wilder AD Jr, Bayne SC, May KN, Studervant JR, Roberson TM. Eighteen-month clinical evaluation of a filled and unfilled dentin adhesive. J Dent 2001; 29: 1-6.

5) Takahashi A, Inoue S, Kawamoto C, Ominato R, Tanaka T, Sato Y, Pereira PRN, Sano H. In vivo long-term durability of the bond to dentin using two adhesive systems. J Adhes Dent 2002; 4: 151-159.

6) Carvalho RM, Tay FR, Sano H, Yoshiyama M, Pashley DH. Long-term mechanical properties of EDTA-demineralized dentin matrix. J Adhes Dent 2000; 2: 193-199.

7) Fuentes V, Ceballos L, Osorio R, Toledano M, Carvalho RM, 
Pashley DH. Tensile strength and microhardness of treated human dentin. Dent Mater 2004; 20: 522-529.

8) Cederlund A, Jonsson B, Blomlof J. Do intact collagen fibers increase dentin bond strength? Swed Dent J 2002; 26: 159-166.

9) Sauro S, Mannocci F, Toledano M, Osorio R, Pashley DH, Watson TF. EDTA or H3PO4/ NaOCl dentine treatments may increase hybrid layers' resistance to degradation: A microtensile bond strength and confocal-micropermeability study. J Dent 2009; 37: 279-288.

10) Yamauti M, Hashimoto M, Sano H, Ohno H, Carvalho RM, Kaga M, Oguchi M, Kubota M. Degradation of resin-dentin bond using $\mathrm{NaOCl}$ storage. Dent Mater 2003; 19: 399-405.

11) Yoshida E, Hashimoto M, Hori M, Kaga M, Sano H, Oguchi H. Deproteinizing effects on resin-tooth bond structures. J Biomed Mater Res B Appl Biomater 2004; 68: 29-35.

12) Pashley DH, Carvalho RM, Sano H, Nakajima M, Yoshiyama M, Shono Y, Fernandes CA, Tay FR. The Microtensile Bond Test: A Review. J Adhes Dent 1999; 1: 299-309.

13) Saboia VPA, Silva FCFA, Nato F, Mazzoni A, Cadenaro M, Mazzoni G, Giannini M, Breschi L. Analysis of differential artificial ageing of the adhesive interface produced by a two-step etch- and-rinse adhesive. Eur J Oral Sci 2009; 117: 618-624.

14) Tay FR, Moulding KM, Pashley DH. Distribution of nanofillers from a simplified-step adhesive in acid-conditioned dentin. J Adhes Dent 1999; 2: 103-117.

15) Hashimoto M, Ohno H, Kaga M, Endo K, Sano H, Oguchi H. In vivo degradation of resin-dentin bonds in humans over 1 to 3 years. J Dent Res 2000; 6: 1385-1391.

16) Koshiro K, Inoue S, Tanaka T, Koase K, Fujita M, Hashimoto $\mathrm{M}$, Sano H. In vivo degradation of resin-dentin bonds produced by a self-etch vs. a total-etch adhesive system. Eur J Oral Sci 2004; 4: 368-375.
17) Yang B, Adelung R, Ludwig K, Bössmann K, Pashley DH, Kern M. Effect of structural change of collagen fibrils on the durability of dentin bonding. Biomaterials 2005; 26: 5021-5031.

18) Carvalho RM, Chersoni S, Frankenberger R, Pashley DH, Parti C, Tay FR. A challenge to the conventional wisdom that simultaneous etching and resin infiltration always occurs in self-etch adhesives. Biomaterials 2005; 26: 1035-1042.

19) Wang Y, Spencer P. Continuing etching of an all-in-one adhesive in wet dentin tubules. J Dent Res 2005; 84: 350-354.

20) Oliveira SS, Marshall SJ, Habelitz S, Gansky SA, Wilson RS, Marshall GW Jr. The effect of a self-etching primer on the continuous demineralization of dentin. Eur J Oral Sci 2004; 112: 376-383.

21) Fukuda R, Yoshida Y, Nakayama Y, Okazaki M, Inoue S, Sano H, Suzuki K, Shintani H, Van Meerbeek B. Bonding efficacy of polyalkenoic acids to hydroxyapatite, enamel and dentin. Biomaterials 2003; 24: 1861-1867.

22) Erhardt MC, Osorio R, Toledano M. Dentin treatment with MMPs inhibitors does not alter bond strengths to cariesaffected dentin. J Dent Res 2008; 12: 1068-1073.

23) Osorio R, Erhardt MCG, Pimenta LAF, Osorio E, Toledano M. EDTA treatment improves resin-dentin bonds' resistance to degradation. J Dent Res 2005; 8: 736-740.

24) Weiss SJ, Slivka A, Wei M. Chlorination of taurine by human neutrophils. Evidence for hypochlorous acid generation. J Clin Invest 1982; 70: 598-607.

25) Olszowski S, Mak P, Olszowska E, Marcinkiewicz J. Collagen type II modification by hypochlorite. Acta Biocimica Polonica 2003; 50: 471-479.

26) Carolina MG, Erhardt A, Osorio R, Toledano M. Dentin treatment with MMPs inhibitors does not alter bond strengths to caries-affected dentin. J Dent 2008; 12: 1068-1073. 